\title{
Arzneimittelpreise \\ aus gesellschaftlicher \\ Perspektive
}

Julian Witte und Wolfgang Greiner

Inhaltsverzeichnis

$1.1 \quad$ Einführung - 4

1.2 Umsetzung einer nutzenbasierten Preisbildung in Deutschland - 5

1.2.1 Paradigmenwechsel mit Einführung des AMNOG - 5

1.2.2 Anwendungserfahrungen des AMNOG - 6

1.3 Determinanten der Preise neuer Arzneimittel - 7

1.3.1 Gesetzlich festgelegte Kriterien zur Preisbildung neuer Arzneimittel - 7

1.3.2 Ergebnisse der nutzenbasierten Preisbildung neuer Arzneimittel - 7

1.4 Ermittlung der gesellschaftlichen Zahlungsbereitschaft für neue Arzneimittel - 10

1.4.1 Wann ist ein Arzneimittelpreis fair? - 10

1.4.2 Faire Preisbildung ohne Einbezug von Kosten-Daten? - 11

1.4.3 Faire Preisbildung von Orphan Drugs - 13

1.5 Ausblick: Neukalibrierung der nutzenbasierten

Preisbildung - 14

Literatur - 15 


\section{- " Zusammenfassung}

Aufgrund der vermehrten Zulassung von Hochpreistherapien ist die mit Einführung der nutzenbasierten Preisbildung in Deutschland vorgesehene Orientierung an Vergleichspreiskorridoren nicht mehr bzw. nur noch sehr eingeschränkt umsetzbar. Der Hintergrund dafür ist, dass diese neuartigen Therapien vielfach keine Vergleichsreferenzen im Markt haben. Diskutiert wird, ob die derzeitigen Preisbildungskriterien und-mechanismen ausreichen, um die Erstattung hochpreisiger Arzneimittel auch zukünftig in einem fairen Interessenausgleich sicherzustellen, oder ob es ergänzender Regulierungsansätze bedarf. Aus gesundheitsökonomischer Perspektive fehlt es vor allem an einer strukturierten Berücksichtigung ökonomischer Evidenz im Rahmen von Bewertung und Preisfindung neuer Arzneimittel. Zudem ist zu erwarten, dass sich die Preisbildung für neue Arzneimittel durch adaptive Preisbildungsmodelle zukünftig stärker in Prozesse verschiebt, die den Verhandlungen nachgelagert sind, um neben der preis- insbesondere die mengenadjustierte Ausgabensteuerung zu ermöglichen. Damit eine solche mengenbezogene Preisadjustierung innerhalb der nutzenbasierten Preisbildungslogik, bestehend aus differenzierten Vergleichstherapien und Teilpopulationen, praktikabel und prozedural fair umsetzbar ist, bedarf es einer verbesserten Datenbasis.

\subsection{Einführung}

Das Jahr 2009 hatte entscheidenden Einfluss darauf, wie Arzneimittelpreise im Jahr 2021 in Deutschland gebildet werden. Innerhalb von zehn Jahren - von 2000 bis 2009 - sind die Arzneimittelausgaben im Durchschnitt jährlich um 5\% und damit im Vergleich zu den übrigen Versorgungssektoren überproportional angestiegen (Beckmann et al. 2010). Der Ausgabenanstieg für die Arzneimittelversorgung war dabei maßgeblich auf patentgeschützte Arzneimittel zurückzuführen (Maag 2010).
Grundlage dieses beobachteten Ausgabentrends waren sowohl Preis- als auch Mengenentwicklungen (Coca et al. 2010). Und obwohl in den vergangenen 40 Jahren preis-, mengenund ausgabenregulierende Instrumente sowohl auf Angebots- als auch Nachfrageseite implementiert wurden, galt die Arzneimittelversorgung im Vergleich zu anderen Versorgungssektoren bis Ende der 2000er Jahre als teilweise schwach reguliert (Gerlinger und Schönwälder 2012). Insbesondere eine Preisregulierung patentgeschützter und damit in der Regel hochpreisiger Arzneimittel war in Deutschland lange Zeit nicht implementiert.

Pharmazeutische Unternehmen nutzen diese Preisfreiheit grundsätzlich, um durch hohe Preise ihre Forschungsinvestitionen zu amortisieren und Gewinne zu maximieren, bevor das Patent ausläuft und der Preiswettbewerb durch Generika einsetzt (Kifman und Neelsen 2010). Gerechtfertigt werden diese hohen Arzneimittelpreise mit hohen Forschungs- und Entwicklungsausgaben in Verbindung mit einem auBerordentlichen Risiko, dass Neuentwicklungen und damit Investitionen scheitern (DiMasi et al. 2016). In vielen Fällen bilden die Preise im deutschen $\mathrm{GKV}^{1}$-Markt auch Maßstäbe für die Preisbildung in Ländern mit einer referenziellen Preisgestaltung (Busse et al. 2015).

Eine Frage, die mittlerweile international als entscheidendes Kriterium für die Preisgestaltung und Erstattung diskutiert wird, ist, ob ein therapeutischer Mehrwert gegenüber bestehenden Therapiealternativen existiert. Deutschland war bis Ende der 2000er Jahre eines der wenigen Länder in der europäischen Union, in denen pharmazeutische Unternehmer Preise neuer Arzneimittel frei festlegen und Produkte auch zu diesem Preis in der GKV-Versorgung ausbieten konnten. Als Anreiz für eine schnelle und vollständige Verfügbarkeit neuer Arzneimittel waren die gesetzlichen Krankenkassen in Deutschland bis zum Jahr 2010 verpflichtet, patentgeschützte Arzneimittel zu diesen Preisen zu erstatten. In den meisten industrialisierten Ländern ha-

1 Gesetzliche Krankenversicherung. 
ben sich hingegen bereits Ende der 1990er bzw. Anfang der 2000er Jahre Prozesse etabliert, welche die Erstattungs- bzw. Verordnungsfähigkeit neuer Arzneimittel in Bezug auf deren Mehrwert bewerten und daraufhin einen strukturierten Preisbildungsprozess initiieren. In Health Technology Assessment-Verfahren (HTA) wird unter Berücksichtigung von Aspekten der medizinischen Wirksamkeit also der Nutzen aus Patientenperspektive und ggf. der Kosten(-Effektivität) überprüft, ob und inwieweit eine neue Gesundheitstechnologie, zum Beispiel ein Arzneimittel, für den Vergütungskatalog eines Sozialversicherungsträgers oder eines nationalen Gesundheitsdienstes geeignet ist (Sorenson et al. 2008). $\mathrm{Zu}$ einem vollständigen HTA gehören auch Aspekte der systematischen und evidenzbasierten sozialen und ethischen Beurteilung des neuen Verfahrens, aber darauf wird national wie international bei Nutzenbewertungen zur Preisfindung kurz nach der Zulassung in der Regel verzichtet. Und obwohl sich international unterschiedliche methodische und ordnungspolitische Ansätze zur nutzenbasierten Preisbildung etabliert haben, ist ihnen ein Ziel gemein: Einen angemessenen und aus Sicht aller beteiligten Parteien fairen Preis für ein Arzneimittel zu finden.

\subsection{Umsetzung einer nutzenbasierten Preisbildung in Deutschland}

\subsubsection{Paradigmenwechsel mit Einführung des AMNOG}

Mit dem Arzneimittelmarktneuordnungsgesetz (AMNOG) im Jahr 2011 hat der Gesetzgeber eine weitreichende Neuregelung der Erstattungsbedingungen für neue Arzneimittel vorgenommen. Eingeführt wurde ein zweistufiges Bewertungs- und Preisbildungsverfahren für neu zugelassene und zunächst auch für bereits im Markt verfügbare Arzneimit- tel, welche noch unter Patentschutz standen und damit nicht vom Festbetragssystem erfasst wurden. Dieses als frühe Nutzenbewertung bezeichnete Verfahren sieht eine unmittelbar auf die Zulassung folgende Bewertung des therapeutischen Zusatznutzens eines neuen Arzneimittels gegenüber dem derzeit verfügbaren Therapiestandard vor. Ein Zusatznutzen soll sich dabei anhand von Verbesserungen patientenrelevanter Endpunkte, zum Beispiel einem verlängerten Überleben oder einer verbesserten Lebensqualität, zeigen. Aspekte wie eine verbesserte Adhärenz oder eine Verhinderung möglicher Folgebehandlungen hingegen sind in der Regel nicht gesonderter Bestandteil des Bewertungsverfahrens, wenn sie nicht durch die patientenrelevanten Endpunkte schon erfasst sind. Anschließend vereinbaren die pharmazeutischen Unternehmer mit dem GKVSpitzenverband einen Erstattungsbetrag. Anders als bei der international vielfach angewandten ,vierten Hürde“ als notwendige Bewertungsvoraussetzung für den Zugang zum Versorgungssystem bleibt der in Deutschland an die arzneimittelrechtliche Zulassung geknüpfte freie Zugang zum GKV-Markt mit der frühen Nutzenbewertung erhalten (Hess 2011). Auch die Preissetzung durch den pharmazeutischen Unternehmer wird von den Neuregelungen des AMNOG nicht tangiert. Der Hersteller bietet ein neues Arzneimittel nach wie vor $\mathrm{zu}$ einem frei festgelegten Abgabepreis an, welcher im ersten Jahr nach Markteinführung auch als Erstattungspreis innerhalb der GKV und PKV fungiert.

Für die sich an die Nutzenbewertung anschließenden Erstattungsbetragsverhandlungen hat der Gesetzgeber den beteiligten Akteuren verpflichtende, aber in der inhaltlichen Ausgestaltung nur orientierende Verhandlungsmaßstäbe an die Hand gegeben. Diese Kriterien sollen einen Interessenausgleich in einem Verhandlungsverfahren gewährleisten und stellen damit den Gegenentwurf zum international verbreiteten Ansatz, auf Basis von Kosten-Effektivitäts-Schwellenwerten Höchstbeträge zu ermitteln, dar. Im Fall eines belegten Zusatznutzen soll sich der 
zu verhandelnde Erstattungsbetrag am Ausmaß des Zusatznutzens, den Jahrestherapiekosten der Vergleichstherapie, den Preisen vergleichbarer Arzneimittel sowie einem europäischen Durchschnittspreis orientieren. Konnte für ein neues Arzneimittel kein Zusatznutzen festgestellt werden, so ist dieses in eine Festbetragsgruppe einzuordnen. Ist für eine entsprechende Höchstbetragsfestlegung keine Festbetragsgruppe verfügbar, so ist ein Erstattungsbetrag $\mathrm{zu}$ vereinbaren, der nicht $\mathrm{zu}$ höheren Kosten als denen der Vergleichstherapie führt. Einigen sich die Parteien nicht auf einen Erstattungsbetrag, wird dieser von einer Schiedsstelle festgesetzt. Dem pharmazeutischen Unternehmer steht es dabei frei, das Bewertungs- und Preisbildungsverfahren jederzeit zu beenden und sein Arzneimittel in Deutschland vom Markt zu nehmen.

\subsubsection{Anwendungserfahrungen des AMNOG}

Ausgewiesenes Ziel des AMNOG war es, Einsparungen im patentgeschützten Arzneimittelsegment zu generieren. Durch die Bewertung des Zusatznutzens und die darauffolgende Verhandlung eines Erstattungsbetrags sollte - so die Idee im Jahr 2010 - das Erstattungsbetragsniveau für neu zugelassene Arzneimittel ebenso wie für patentgeschützte Präparate des Bestandsmarkts an ein zum damaligen Zeitpunkt niedrigeres, europäisches Preisniveau angeglichen werden. Der Gesetzgeber bezifferte dieses Preissenkungspotenzial in der Gesetzesbegründung des AMNOG auf durchschnittlich $16 \%$ (Bundestags-Drucksache 2010). Seitdem wurde mehrfach kritisiert, dass das AMNOG trotz seiner klaren Zielformulierung in praxi nur unzureichende Einsparungen generiert (Bundestags-Drucksache 2013). Empirische Beobachtungen deuten jedoch in eine andere Richtung: Der durchschnittliche Nutzenbewertungsrabatt liegt seit 2012 relativ stabil bei knapp über $20 \%$. Diese Rabatte realisieren ein in den vergangenen Jahren konstant wachsen- des Einsparvolumen für die GKV. Zu den bislang daraus erzielten Einsparvolumina innerhalb der GKV-Versorgung liegen unterschiedliche, jedoch hinsichtlich der Größenordnung vergleichbare Angaben vor. Je nach Quelle belief sich das Einsparvolumen im Jahr 2017 auf 1,6 bis 1,8 Mrd. €. Für 2020 wurde ein weiterer Anstieg der Einsparungen durch Erstattungsbeträge auf 3,9 Mrd. $€$ berichtet (Maag 2021).

Zehn Jahre nach Einführung des AMNOG ist das Verfahren etabliert und unter allen Verfahrensbeteiligten im Grundsatz akzeptiert. Der Prozess der frühen Nutzenbewertung wird von relevanten Stakeholdern übergreifend als transparent, fair und wissenschaftlich fundiert beschrieben (Greiner et al. 2020). Bis Ende 2019 haben 259 Arzneimittel mit neuem Wirkstoff in 439 Verfahren eine frühe Nutzenbewertung und daran anschließende Erstattungsbetragsverhandlungen durchlaufen. Dass $87 \%$ dieser Wirkstoffe Mitte 2020 noch in Deutschland verfügbar sind und für $82 \%$ aller Erstattungsbeträge eine Verhandlungslösung (und keine Festsetzung durch eine Schiedsstelle) gelang, wird als Indikator für ein hoch funktionales, weil ,lernendes“ System gewertet (Hecken 2020), das sogar im internationalen Kontext als Blaupause für ein nutzenbasiertes Preisbildungssystem dient (McGrail 2020).

Zuletzt ist jedoch durch den Marktzugang extrem hochpreisiger Gentherapien (Korzilius 2019; Hecken 2019) sowie Mengen- (und damit Kosten-)Steigerungen in der Onkologie (Sussell et al. 2019; Ärzteblatt 2017) die finanzielle Belastungsfähigkeit des Gesundheitssystems in Frage gestellt worden (Korzilius 2019; Danzon 2018). Dabei lag der Anteil der Arzneimittelausgaben an den gesamten GKVAusgaben mit 16,3\% im Jahr 2019 im Verhältnis zu den Vorjahren weitestgehend auf vergleichbarem Niveau (Bundesministerium für Gesundheit 2020). Dennoch wird die Angemessenheit von Arzneimittelpreisen (Morgan et al. 2020) und die Funktionalität des deliberativen, nutzenbasierten Preisbildungsverfahren des AMNOG zur Bildung fairer Preise an- 
gezweifelt (Laschet 2020). Es wird diskutiert, ob die vom Gesetzgeber vorgegebenen Preisbildungskriterien allein noch geeignet sind, auch zukünftig ,wirtschaftliche und kosteneffiziente“" (Bundestags-Drucksache 2010) Arzneimittelpreise zu bilden.

\subsection{Determinanten der Preise neuer Arzneimittel}

\subsubsection{Gesetzlich festgelegte Kriterien zur Preisbildung neuer Arzneimittel}

Das Ausmaß des vom Gemeinsamen Bundesausschuss (G-BA) im Rahmen der Nutzenbewertung festgestellten Zusatznutzens ist das zentrale Preisbildungskriterium neuer Arzneimittel. Gemäß $\S 130$ b Abs. 9 SGB V i. V.m. $\S \S 5$ und 6 der Rahmenvereinbarung nach $\S 130 \mathrm{~b}$ Abs. 8 SGB V (RahmenV) sind unter freier Würdigung aller Umstände des Einzelfalls und unter Berücksichtigung der Besonderheiten des jeweiligen Therapiegebietes zusätzlich drei weitere Kriterien heranzuziehen:

- Der Beschluss des G-BA über die Nutzenbewertung nach $\S 35 \mathrm{a}$ Abs. 3 SGB V mit den darin getroffenen Feststellungen, insbesondere

- zum Zusatznutzen des Arzneimittels im Verhältnis zur zweckmäßigen Vergleichstherapie (zVT),

- zur Anzahl der Patientinnen und Patienten bzw. zur Abgrenzung der für die Behandlung in Frage kommenden Patientengruppen,

- zu Anforderungen an eine qualitätsgesicherte Anwendung und

- zu den Therapiekosten auch im Vergleich zur zVT.

- Die Jahrestherapiekosten vergleichbarer Arzneimittel

- Die von dem pharmazeutischen Unternehmer gemäß $\S 3$ Abs. 2 mitgeteilten tatsäch- lichen Abgabepreise in anderen europäischen Ländern.

Dabei können die Vertragsparteien nicht abweichend vom Beschluss des G-BA von einem anderen, höheren oder niedrigeren Zusatznutzen ausgehen. Da jedoch keine algorithmischen Vorgaben zur Monetarisierung eines bestimmten Zusatznutzens oder eine festgesetzte Skalierung der Zusatznutzenausmaße zueinander existieren ${ }^{2}$, unterliegt dieser Parameter einer Verhandlungslösung. Das Ausmaß des Zusatznutzens ist in jedem Preisbildungsverfahren neuer Arzneimittel heranzuziehen. Hingegen sind die Jahrestherapiekosten der zVT bzw. die Kosten vergleichbarer Arzneimittel sowie die europäischen Abgabepreise nur bei Vorliegen eines zumindest in Teilen des gegenständlichen Anwendungsgebiets belegten Zusatznutzens als Preisbildungskriterien Verhandlungsgegenstand. Dabei sind o. g. Kriterien nur bedingt deterministisch, sondern unterliegen regelhaft einer Verhandlungseinigung. Insbesondere die relative Gewichtung dieser Kriterien dürfte fortwährend Gegenstand der Verhandlungen sein. Dies gilt auch für weitere verfahrensrelevante Aspekte wie die Größe des Anwendungsgebietes, das Wettbewerbsumfeld (z. B. absehbare Generikaeintritte), in der Vergangenheit abgeschlossene Schiedsverfahren oder die Qualität der verfügbaren Evidenz.

\subsubsection{Ergebnisse der nutzenbasierten Preisbildung neuer Arzneimittel}

Zur Bewertung der Preisbildungsergebnisse neuer Arzneimittel können verschiedene Ergebnismaße herangezogen werden. Bei einem Arzneimittel, das einen Zusatznutzen gegenüber der zVT aufweist, wird der Erstattungs-

2 BSG, 04.07.2018 - B 3 KR 20/17 R Rn. 26; BSG, 04.07.2018 - B 3 KR 21/17R Rn. 20; BSG, 04.07.2018 - B 3 KR 21/17 R Rn. 37. 
betrag durch einen Zuschlag auf die Jahrestherapiekosten der zVT verhandelt. In den Fällen ohne belegten Zusatznutzen soll der Erstattungsbetrag nicht zu höheren Jahrestherapiekosten als die zVT führen.

Der durchschnittliche Preisabschlag auf den Markeintrittspreis eines neuen Arzneimittels beträgt für alle bis Ende 2019 erstbewerteten Arzneimittel mit abgeschlossenen Erstattungsbetragsverhandlungen 21,7\% (Median: 19,7\%, SD 3 : 14,1; Greiner et al. 2020). Für das Verständnis der Preisbildungsergebnisse nach erfolgter Nutzenbewertung ist die Unterscheidung der Preisbildungsarithmetik von entscheidender Bedeutung. Wird in Folge eines positiven Bewertungsergebnisses „frei“ verhandelt oder ,muss“ (bzw. ,,soll“"4) sich die Preisfindung in Folge nicht belegter therapeutischer Überlegenheit an den Therapiekosten der zVT orientieren? Empirisch zeigt sich, dass in deterministischen Preisbildungsverfahren für Wirkstoffe ohne belegten Zusatznutzen $(n=77)$ die Chance für einen Preisabschlag von über $30 \%$ auf den Listenpreis gegenüber „freien“ Verhandlungsverfahren nach belegtem Zusatznutzen $(\mathrm{n}=145)$ um das Fünffache erhöht ist $\left(\mathrm{OR}^{5}=4,86, \mathrm{p}=0,001\right)$. Der durchschnittliche Abschlag auf den Markeintrittspreis liegt bei Wirkstoffen mit belegtem Zusatznutzen mit 19,1\% (Median: 17,7\%, SD: 10,8) demzufolge deutlich unterhalb des mittleren Preisabschlags von Wirkstoffen ohne belegten Zusatznutzen (26,4\%, Median: 24,3\%, SD: 17,9).

Werden die beobachteten Preisabschläge im Zeitverlauf kumuliert und über die Verfahrensanzahl gemittelt, wird ersichtlich, dass sich die verhandelten oder festgesetzten Preisabschläge inzwischen auf stabilem Niveau etabliert haben. Nach 110 abgeschlossenen Erstattungsbetragsverhandlungen bzw. Preisbildungsverfahren ab Ende 2016 liegt der mittlere Preisabschlag konstant zwischen 20,0\%

3 Standardabweichung (SD, englisch Standard Deviation).

4 Seit Inkrafttreten des Arzneimittelversorgungsstärkungsgesetz (AMVSG) im März 2017.

5 Odds-Ratio. und $21,3 \%$. Zudem konvergieren die mittleren Preisabschläge für Wirkstoffe mit belegtem und nicht belegtem Zusatznutzen in der Erstbewertung. - Abb. 1.1 zeigt, dass sich ein Preisabschlagskorridor zwischen $18 \%$ und $27 \%$ etabliert hat, was sich durch die gestiegene Anwendungserfahrung der Vertragsparteien erklären ließe. Außerdem könnte es ein Hinweis darauf sein, dass den Erstattungsbetrag begleitende vertragliche Vereinbarungen, beispielsweise mengenbezogene Preisstaffelungen oder Pay-for-Performance-Bestandteile, eine zunehmende Rolle einnehmen. ${ }^{6}$

Die absolute Höhe des Rabatts auf den Markteintrittspreis eines neuen Arzneimittels ist jedoch nur begrenzt als Bewertungskriterium für die Preisbildung geeignet, da der Rabatt umso höher ausfällt, je höher der pharmazeutische Unternehmer den Listenpreis beim erstmaligen Inverkehrbringen festlegt hat. Praktisch ist der Erstattungsbetrag eines neuen Arzneimittels ein Aufschlag auf die Kosten der zVT (Bottom-Up-Prinzip). Ausgenommen davon sind Orphan Drugs (bis zur Überschreitung einer Umsatzschwelle); für diese beschließt der G-BA über das Ausmaß des Zusatznutzens ohne Festlegung einer zVT. Das Bottom-Up-Prinzip ist für Orphan Drugs deshalb nicht anwendbar. Stattdessen erfolgt die Preisbildung hier im Top-Down-Prinzip ausgehend vom frei festgelegten Listenpreis.

Der durchschnittliche Aufschlag auf die Jahrestherapiekosten der zVT beträgt bislang das 2,8-Fache der Jahrestherapiekosten der zVT (• Tab. 1.1). Erwartungsgemäß sind diese bedeutend höher, wenn der G-BA einen $\mathrm{Zu}$ satznutzen festgestellt hat. Für Wirkstoffe mit belegtem Zusatznutzen wurde bis Ende 2019 im Durchschnitt ein Preisaufschlag in Höhe des Fünffachen der Jahrestherapiekosten der zVT vereinbart oder festgesetzt. Zwischen den

6 Ein Indiz dafür ist unter anderem, dass der GKV-Spitzenverband zuletzt für den Wirkstoff Darvadstrocel erstmals auf seiner Webseite darüber Auskunft gegeben hat, dass er im Rahmen der Erstattungsbetragsverhandlungen mit dem pharmazeutischen Unternehmer eine Vereinbarung über eine erfolgsabhängige Vergütung geschlossen hat. 


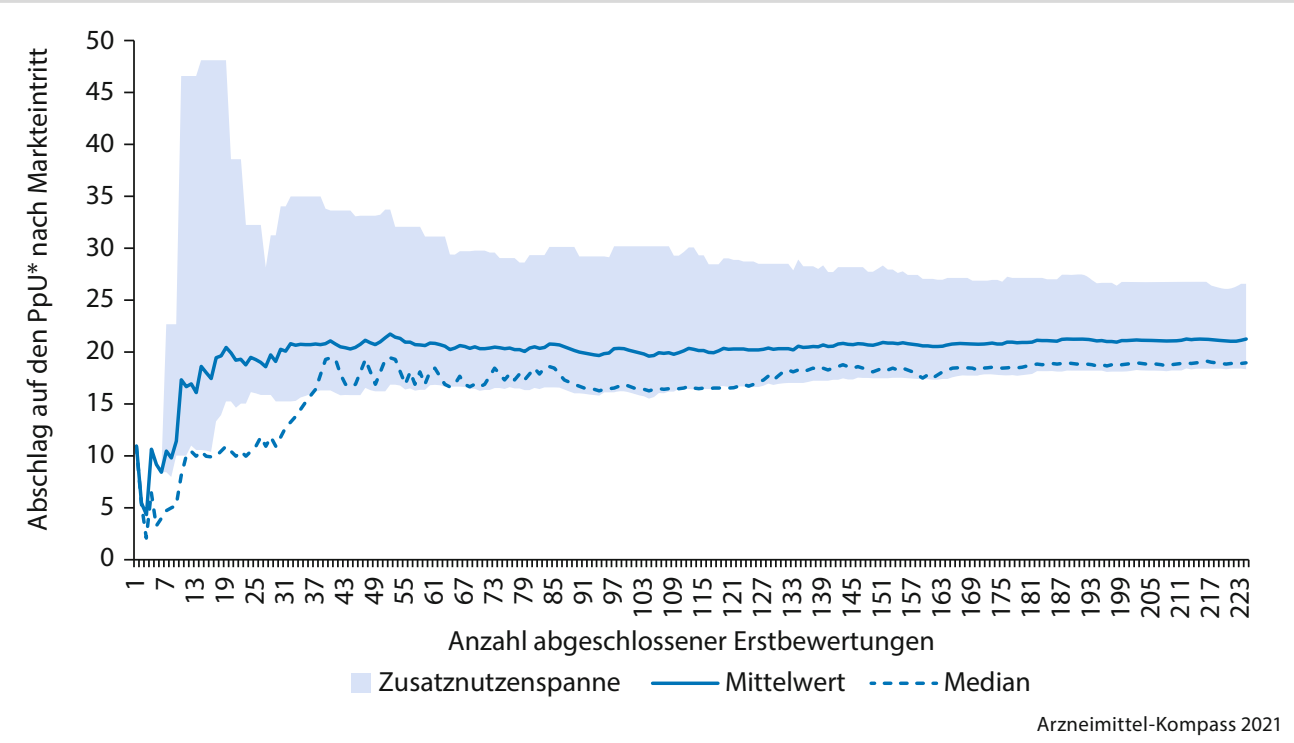

- Abb. 1.1 Entwicklung des mittleren Preisabschlags nach Erstbewertung im Zeitverlauf. *PpU = Preis des pharmazeutischen Unternehmers, in \% (Quelle: Eigene Auswertung und Darstellung, Stand: 01.04.2020)

- Tab. 1.1 Preisaufschläge auf die Jahrestherapiekosten der zVT

\begin{tabular}{|l|l|l|c|c|}
\hline Bewertungsergebnis & N & Mittelwert in \% & Spanne in \% & SD \\
\hline $\begin{array}{l}\text { Zusatznutzen belegt } \\
\text { Gesamt }\end{array}$ & 69 & 505 & $0-6.368$ & 1.125 \\
\hline $\begin{array}{l}\text { Stand: } 01.04 .2020 \\
\text { Arzneimittel-Kompass 2021 }\end{array}$ & 139 & 280 & $-77-6.368$ & 840 \\
\hline
\end{tabular}

positiven Zusatznutzenausprägungen ist deskriptiv ebenfalls ein signifikanter Unterschied in den mittleren Preisaufschlägen auf die zVT zu beobachten (Greiner et al. 2020).

Unabhängig von den relativen Ergebnismaßen zur Beschreibung der Erstattungsbetragsverhandlungen kann es aus Systemperspektive auch von Interesse sein, die absolute Höhe der Jahrestherapiekosten neuer Arzneimittel nach Abschluss des Preisbildungsverfahren $\mathrm{zu}$ betrachten. Im Durchschnitt fallen für ein nutzenbewertetes Arzneimittel nach Abschluss der Erstattungsbetragsverhandlungen zwischen 2011 und 2020 Jahrestherapiekos- ten in Höhe von knapp unter $100.000 €$ pro Patientin/Patient an (- Tab. 1.2). Dabei sind zusätzliche Kosten durch die Anwendung als Kombinationstherapie berücksichtigt. Arzneimittel mit belegtem Zusatznutzen erlösen dabei gegenüber solchen ohne belegten Zusatznutzen nahezu doppelt so hohe Jahrestherapiekosten, für nutzenbewertete Orphan Drugs fallen fast viermal höhere Jahrestherapiekosten an als für Non-Orphan Drugs. Dabei kann empirisch beobachtet werden, dass bei Orphan Drugs der Spielraum für Preisnachlässe auf den Markteintrittspreis zwar grundsätzlich etwas größer ist, sich mit einem im Zeitverlauf 
- Tab. 1.2 Höhe der Jahrestherapiekosten nutzenbewerteter Arzneimittel unter Erstattungsbetrag

\begin{tabular}{|l|l|l|l|l|}
\hline Konstellation & N & Mittelwert in $€$ & Spanne in $€$ & SD \\
\hline Zusatznutzen belegt & $\mathbf{1 4 5}$ & $\mathbf{1 1 8 . 4 0 7}$ & $\mathbf{4 5 - 9 4 6 . 9 6 1}$ & $\mathbf{1 6 2 . 0 0 5}$ \\
\hline $\begin{array}{l}\text { Zusatznutzen nicht belegt } \\
\text { Gesamt }\end{array}$ & 71 & 56.281 & $75-466.849$ & 107.267 \\
\hline $\begin{array}{l}\text { Orphan Drug } \\
\text { Non Orphan Drug }\end{array}$ & 68 & 976.986 & $45-946.961$ & 149.172 \\
\hline $\begin{array}{l}\text { Stand: 01.04.2020 } \\
\text { Arzneimittel-Kompass 2021 }\end{array}$ & 148 & 52.640 & $7.475-946.961$ & 199.404 \\
\hline
\end{tabular}

zunehmenden Anstieg der jährlichen Behandlungskosten jedoch nicht vergrößert (Greiner et al. 2020).

\subsection{Ermittlung der gesellschaftlichen Zahlungsbereitschaft für neue Arzneimittel}

\subsubsection{Wann ist ein Arzneimittelpreis fair?}

Ziel des AMNOG ist es, einen Interessenausgleich zwischen pharmazeutischem Unternehmer und GKV-Spitzenverband über eine angemessene Höhe der Jahrestherapiekosten eines neuen Arzneimittels im Verhältnis zum vom G-BA festgestellten Nutzen bzw. Zusatznutzen zu erreichen. Vertreter der gesetzlichen Krankenkassen betonen hingegen regelhaft, dass sich aus der derzeit in Deutschland angewandten nutzenbasierten Preisbildung keine fairen oder gerechten Preise ergeben (Jablonka et al. 2021). Allerdings ist nicht klar definiert, $a b$ welcher Höhe Arzneimittelpreise als fair bzw. gerecht zu bewerten sind. Unter ordnungspolitischen Gesichtspunkten könnte ein gerechter Preis als Wettbewerbspreis definiert werden. Einen echten Wettbewerbspreis kann es allerdings aufgrund der beschriebenen Besonder- heiten des Arzneimittelmarktes für patentgeschützte Arzneimittel nicht geben (Schlander et al. 2012).

Grundsätzlich liegt die Zahlungsbereitschaft der GKV nach Markteintritt eines neuen Arzneimittels oberhalb der marginalen Produktionskosten des entsprechenden Produkts. Damit sich ein freier Preiswettbewerb zwischen pharmazeutischen Unternehmern nicht innovationsfeindlich auswirkt, garantieren $\mathrm{Pa}$ tente und Schutzzertifikate ein zeitlich begrenztes Angebotsmonopol. Anreize für innovative Behandlungskonzepte, die nachweislich die gesundheitliche Situation der Patientinnen und Patienten verbessern, sind vor dem Hintergrund vieler nach wie vor nicht bzw. nur eingeschränkt behandelbarer Erkrankungen gesellschaftlich erwünscht. Gleichzeitig wird unter Berücksichtigung hoher Markteintrittspreise neuer Arzneimittel die Funktionalität dieses Verfahrens zur Ermittlung fairer Preise in Frage gestellt und stattdessen bei den pharmazeutischen Unternehmern eine Ausnutzung eben dieser Vermarktungsprivilegien vermutet. Auch aus diesem Grund wird die zwölfmonatige Phase freier Preissetzung von Krankenkassenvertretern kritisch hinterfragt (Korzilius 2019). Dies offenbart jedoch zwei Probleme: So realisiert ein Arzneimittel nur aufgrund eines hohen Preises noch keine hohen Kosten für das GKV-System, obschon Ausstrahlungseffekte auf zukünftige Preissetzungen berücksichtigt werden müssen. Schwe- 
rer wiegt jedoch, dass in Deutschland keine offene gesellschaftliche Diskussion darüber geführt wurde, wie hoch die Zahlungsbereitschaft für neue Arzneimittel ist bzw. wie mit Opportunitäten $\mathrm{zu}$ verfahren ist. Im wissenschaftlichen Kontext ist die Bestimmung der gesellschaftlichen Zahlungsbereitschaft aus einer Aggregation der individuellen Zahlungsbereitschaften etabliert, zum Beispiel durch Befragungen. Aufgrund der politischen Sorge vor impliziter Rationierung werden in der Diskussion über faire Arzneimittelpreise entsprechende Überlegungen jedoch nicht einbezogen (Greiner 2020). Erschwerend kommt hinzu, dass Zahlungsbereitschaften für die in der frühen Nutzenbewertung sehr unterschiedlichen Ausprägungen des Zusatznutzens (z. B. symptomfreie Tage oder Verzögerung von Rezidiven) nur schwer zu ermitteln sind. Für ein universelles, eindimensionales Maß - wie etwa die sogenannten qualitätskorrigierten Lebensjahre (QALY, englisch quality-adjusted life years) - ist dies deutlich leichter $\mathrm{zu}$ erreichen (Wasem 2018).

\subsubsection{Faire Preisbildung ohne Einbezug von Kosten-Daten?}

Der Gesetzgeber hat sich mit Einführung des AMNOG letztlich dazu entschieden, das Austarieren der Zahlungsbereitschaft nicht auf gesellschaftlicher Ebene, sondern deliberativ zwischen pharmazeutischem Unternehmer und GKV-Spitzenverband aushandeln zu lassen (Sussex et al. 2013). Die Diskussion um die Zahlungsbereitschaft für neue Arzneimittel ist inzwischen weitestgehend einer Diskussion um die Zahlungsfähigkeit gewichen. Dies lässt sich unter anderem daraus ableiten, wie unterschiedlich Vertreter des GKV-Spitzenverbandes die Ergebnisse der Erstattungsbetragsverhandlungen im Zeitverlauf bewerten: Nach circa 20 abgeschlossenen Preisverhandlungen bezeichnete das damalige Vorstandsmitglied Johann-Magnus von Stackelberg das AMNOG noch als einen „Riesenerfolg“, wobei nicht unmittelbar die Höhe des verhandelten Erstat- tungsbetrages, sondern erwartbare Einsparungen über den Lebenszyklus nutzenbewerteter Arzneimittel Grund dieser positiven Bewertung waren (o. V. 2013). Inzwischen wird jedoch regelhaft vor einer Überforderung des GKV-Systems infolge zu hoher Arzneimittelpreise gewarnt. Dabei steht auch die Frage im Mittelpunkt, ob das AMNOG-Verfahren (noch) zur Preisregulierung neuer Arzneimittel geeignet ist (Jablonka et al. 2021).

Denn die zusätzliche Zulassung therapeutischer Solisten für neue Therapien bei onkologischen und/oder Orphan-Indikationen, d. h. in bereits etablierten Hochpreisindikationen, vergrößert das medikamentös behandelbare Patientenkollektiv. Für die GKV problematisch ist, dass in diesen Konstellationen trotz AMNOGinduzierter Reduktion des Erstattungsbetrags ein hoher Budgeteffekt entsteht, da keine Substitution bestehender Therapien erfolgt. Die Kosten der neuen Therapie fallen somit primär additiv an. Allerdings werden bislang auch nicht alle AMNOG-Instrumente, insbesondere die Kosten-Nutzen-Bewertung herangezogen, um ein möglichst vollständiges Bild der Effekte eines neuen Arzneimittels in der Preisfindung zu erlangen. Kosten-Nutzen-Bewertungen sind indes kein Instrument zu einer schnell wirksamen Ausgabenbegrenzung und werden in Deutschland auch nicht neu thematisiert. Seit Inkrafttreten des GKV-Wettbewerbsstärkungsgesetzes im Jahr 2004 sieht das SGB V vor, dass grundsätzlich eine Bewertung des Kosten-Nutzen-Verhältnisses stattfinden kann. Im AMNOG ist diese Bewertung jedoch erst am Ende des Verfahrensprozesses nach gescheiterten Schiedsverfahren - vorgesehen. Dann sind in der Regel schon 15 Monate seit Markteinführung eines neuen Arzneimittels vergangen. Nach aktueller Gesetzeslage können mindestens zwei weitere Jahre bis zum Ergebnis einer Kosten-Nutzen-Analyse durch das Institut für Qualität und Wirtschaftlichkeit im Gesundheitswesen (IQWiG) vergehen. Zudem sind die derzeit geltenden methodischen Konzepte des IQWiG zur Durchführung einer Kosten-Nutzen-Bewertung umstritten und international nur teilweise anschlussfä- 
hig (Greiner et al. 2010). Darum ist der Anreiz zu einer Kosten-Nutzen-Bewertung nach einer Schiedsstellenentscheidung nicht hoch. Wohlfahrtsmaximal ist die Lösung jedoch aller Voraussicht nach nicht, weshalb auch der Sachverständigenrat zur Begutachtung der Entwicklung im Gesundheitswesen (SVR-Gesundheit) in seinem Gutachten 2014 anregte, im Rahmen der Preisverhandlungen Ergebnisse aus Kosten-Nutzen-Bewertungen fakultativ einzubeziehen (Sachverständigenrat zur Begutachtung der Entwicklung im Gesundheitswesen 2014).

Politisch hingegen scheint eine verpflichtende Kosten-Nutzen-Bewertung für alle neuen Arzneimittel derzeit kaum durchsetzbar zu sein (o. V. 2020a). Gegen einen solchen vollständigen Paradigmenwechsel spricht auch die hohe Akzeptanz und bisherige Flexibilität des AMNOG-Verfahrens. Insbesondere von Seiten einiger Kostenträger mehren sich jedoch die Stimmen, diese Daten zumindest in bestimmten Verfahrenskonstellationen (z. B. bei innovativen sehr hochpreisigen Therapien) fakultativ oder sogar obligatorisch in die Verfahren einzubringen. $\mathrm{Ob}$ und wann es überhaupt zu einer Etablierung des Instruments kommt, bleibt abzuwarten. So betonte der GKV-Spitzenverband, dass erst andere Maßnahmen ausgeschöpft werden sollten, beispielsweise die Erstattungsfähigkeit bei bestimmten Indikationen von einem belegten $\mathrm{Zu}$ satznutzen abhängig zu machen, bevor die Einbeziehung von Kosten-Nutzen-Bewertungen erwogen wird (o. V. 2020a).

Zwar beantwortet auch die Kosten-NutzenAnalyse nicht alle Fragen zur Vorteilhaftigkeit neuer Arzneimittel, insbesondere, solange keine gesellschaftlich akzeptierten Schwellenwerte für die Erstattungshöhe vorliegen. Dennoch hätte man unter Opportunitätskostenaspekten eine zusätzliche Information, um Innovationen adäquat aus gesellschaftlicher Perspektive zu bewerten (Cassel und Ulrich 2016). Aus den Erfahrungen mit dem zum AMNOG-Verfahren vergleichbaren HTA-Verfahren in Frankreich kann man ableiten, dass es zum Austarieren der Qualitätsstandards ei- ner gewissen Übergangsperiode bedürfen wird (Toumi et al. 2017).

Vor dem Hintergrund der Debatte um Hochpreistherapien würde sich auch eine im internationalen Kontext übliche transparente Auseinandersetzung mit dem potenziellen Budgeteffekt (Budget Impact) der Erstattung einer neuen Therapie anbieten (Goldstein und Sarfaty 2016). Es ist zwar davon auszugehen, dass durch die obligatorische Berücksichtigung mengenbezogener Aspekte im Rahmen der Erstattungsbetragsbildung bereits heute indirekt auch Budget-Überlegungen in den Preisbildungsprozess neuer Arzneimittel einfließen, allerdings böten gut durchgeführte Budget Impact-Analysen den Vorteil, den Einfluss von zum Beispiel zentralen Annahmen zur zukünftigen Marktdurchdringung eines neuen Arzneimittels transparenter als heute zu simulieren und in die Preisfindung einzubeziehen. Solche Analysen sind beispielsweise in den Niederlanden, in Polen oder in Belgien obligatorischer Verfahrensbestandteil. Zudem existieren verschiedene Guidelines für deren standardisierte Erstellung, sodass eine schnelle Umsetzung im deutschen Versorgungskontext möglich wäre.

Darüber hinaus zeigt sich im Rahmen von Analysen der Preisbildungsergebnisse eine hohe Sensitivität des AMNOG-Verfahrens für die Evidenzqualität. Diese könnte zukünftig durch eine verbesserte Nutzung der bestehenden rechtlichen Möglichkeiten für adaptive Erstattungsmodelle, zum Beispiel Pay-forPerformance- oder mengenbezogene Verträge, stärker als heute Einfluss auf den Preisbildungsprozess nehmen. Dabei sind gleichwohl Probleme bei der praktischen Umsetzung offenkundig. Neben der unterschiedlichen Erstattung von Arzneimitteln im ambulanten und stationären Sektor sind die insbesondere für die Umsetzung adaptiver Preismodelle erforderlichen Daten derzeit nicht in vollem Umfang verfügbar (Stegmaier 2019). Hierzu gehören die stationären Leistungsdaten zur Abbildung des dortigen Einsatzes neuer Arzneimittel und die Versorgungsdaten zum Beispiel auf Basis der GKV-Abrech- 
nungsdaten. Zudem liegen Vorschläge vor, Erstattungsbeträge zukünftig dynamisch an die verfügbare Evidenz zu knüpfen, zum einen durch dynamische Evidenzpreise (o. V. 2020b), also Preise, die sich im Lebenszyklus eines Arzneimittels an sich verändernde Evidenzlagen anpassen, zum anderen durch die Einführung zeitlich begrenzter vertraulicher Preisabschläge (Greiner et al. 2020).

Es gibt darüber hinaus Diskussionen, die allokative Effizienz des AMNOG-Verfahrens dadurch zu verbessern, den verhandelten Erstattungsbetrag bereits auf den ersten Tag ab Marktverfügbarkeit rückwirken zu lassen (GKV-Spitzenverband 2019). Dabei konnten Analysen im Jahr 2016 zeigen, dass sich durch eine entsprechende Rückwirkung kaum nennenswerte Einsparungen generieren lassen, weil die Marktdurchdringung der allermeisten Arzneimittel erst einmal langsam anläuft. Entsprechende Vorschläge scheiterten (bislang) auch an juristischen Erwägungen wie einem Rückwirkungsverbot sowie an der Notwendigkeit verlässlicher Rahmenbedingungen für pharmazeutische Unternehmer zum Zeitpunkt des Markteintritts. Bei einer vollständigen Rückwirkung des Erstattungsbetrags entstünde eine nur begrenzt antizipierbare Unsicherheit über den tatsächlichen Preis des neuen Arzneimittels zum Zeitpunkt des Markteintritts. In der Konsequenz müssten Hersteller innerhalb des ersten Jahres Rücklagen in Höhe eines beträchtlichen Anteiles des Produktumsatzes bilden. Über den Markteintritt müsste zudem ohne jeden Anhaltspunkt über den Preis, der erst am Ende des Bewertungsprozesses festgelegten wird, entschieden werden, was aus ordnungspolitischer Sicht zumindest fragwürdig ist.

\subsubsection{Faire Preisbildung von Orphan Drugs}

Die Bildung angemessener und fairer Erstattungsbeträge für Orphan Drugs ist hinsichtlich weitestgehend referenzfreier und damit vielfach als arbiträr (Picavet et al. 2014) be- schriebener Markteintrittspreise ein komplexer Prozess. Kritisiert wird, dass politisch gewährte Forschungs- und Entwicklungsanreize wie eine beschleunigte und kostenreduzierte $\mathrm{Zu}$ lassung sowie der Verzicht auf eine uneingeschränkte Nutzenbewertung durch den G-BA Orphan Drugs zu einem aus Sicht der Industrie lukrativen Geschäftsmodell machen. Das wiederum fördert nicht-nutzenadäquate Preise, die auch nicht durch Erstattungsbetragsverhandlungen korrigiert werden können (ArzneiTelegramm 2017). Zentraler Grund dafür sei, dass dem GKV-Spitzenverband das Verhandlungsmandat für bedeutend höhere Preisabschläge fehle.

Obwohl die Preisbildung für Orphan Drugs der gleichen ökonomischen Logik wie der Preisbildung für Non-Orphan Drugs folgt, führt das Angebotsmonopol bei Orphan Drugs zunächst aus denselben Gründen wie bei NonOrphan Drugs (Marktexklusivität, Erlösmaximierung durch den Unternehmer, fehlender Preiselastizität der Nachfrager) zu hohen Abgabepreisen. Bei Orphan Drugs wirkt jedoch zusätzlich preiserhöhend, dass die Nachfrage nicht nur preisunelastisch, sondern aufgrund der Seltenheit der zu behandelnden Erkrankungen stark limitiert ist (Simoens 2011). Hinzu kommt der Vorwurf, dass durch strategische Zulassungsanträge der pharmazeutischen Unternehmer eine „Orphanisierung“ vieler Erkrankungen angestrebt werde, um jeweils in vielfach monopolistischer Marktkonfiguration höhere Preise erzielen zu können (Simoens 2011). Demgegenüber ist zu berücksichtigen, dass es in der EU keine Zulassungsbegrenzung für eine Orphan-Indikation gibt, also mehrere Arzneimittel für die Behandlung eines seltenen Leidens zugelassen werden können. Infolgedessen kann das Angebotsmonopol eines Orphan-Medikaments auch durch die Verfügbarkeit anderer Produkte aufgehoben und zumindest in Teilen ein Preiswettbewerb initiiert wird. So gibt es beispielsweise für das fortgeschrittene Nierenzellkarzinom und die zystische Fibrose jeweils mehrere inzwischen auch nutzenbewertete Arzneimittel mit ausgewiesenem Orphan-Status (Greiner und Witte 2017). 
Für First-in-Class-Orphan Drugs erscheint insofern ein Marktzugang und eine Preisfindung auf Basis placebokontrollierter oder noch schwächerer Studiendesigns grundsätzlich noch angemessen. Sobald Therapiealternativen verfügbar sind, ist indes nicht ersichtlich, warum nicht auch Orphan Drugs in Relation zu ebendiesen bewertet und in der Folge einer komparativ-nutzenbasierten Preisbildung unterzogen werden sollten (Vgl. für diesen Vorschlag Greiner und Witte 2018). Analysen der Preisbildungsergebnisse aus Frankreich zeigen, dass eine entsprechende komparatorbasierte Preisbildung auch für Orphan Drugs möglich ist. In aktuellen Untersuchungen konnte gezeigt werden, dass vor allem die Verfügbarkeit alternativer Behandlungsmöglichkeiten neben dem Ausmaß des Zusatznutzens, der Art des Komparators in der zulassungsrelevanten klinischen Studie sowie dem Datum der Markteinführung einen relevanten Einfluss auf die Erstattungshöhe hat (Korchagina et al. 2017; Korchagina et al. 2015).

Solche vergleichenden Preispunkte werden für die nutzenbasierte Preisbildung von Orphan Drugs im AMNOG-Prozess derzeit nicht (transparent) herangezogen. Vielmehr deuten die bisherigen Erstattungsbeträge für neue Orphan Drugs darauf hin, dass die Zahlungsbereitschaft der GKV in diesem Marktsegment vergleichsweise hoch und das Absenkungspotential des Markteintrittspreises des pharmazeutischen Unternehmers vergleichsweise gering ist. Daraus jedoch auf eine vollständige Entkopplung der Orphan Drug-Preisbildung von den Grundsätzen der nutzenbasierten Preisbildung zu schließen, wäre nicht sachgerecht. Vielmehr lässt sich nach unseren Berechnungen im Rahmen multivariater Analysen ein Einfluss der Evidenzqualität bei Markteintritt - abgebildet über einen befristeten Nutzenbewertungsbeschluss - auf die Erstattungsbetragshöhe identifizieren. Durch die im Jahr 2019 vom Gesetzgeber eingeführte anwendungsbegleitende Datenerhebung, die zukünftig mit weiteren initialen Preisabschlägen verbunden sein dürfte, wird die bereits gelebte Praxis auch gesetzlich geregelt. Es ist davon auszugehen, dass damit insbesondere die unsichere Evidenzlage von Orphan Drugs adressiert wird. Um negative Innovationsanreize zu vermeiden, schlug der unparteiische Vorsitzende des G-BA, Professor Hecken, jüngst vor, entsprechende initiale Preisabschläge parallel zur Evidenzgenerierung vertraulich $\mathrm{zu}$ halten.(Greiner et al. 2020) Gleichzeitig wird die Interpretation der nach der Nutzenbewertung erhobenen Versorgungsdaten und deren Einbindung in die Preisverhandlungssystematik eine der größeren Herausforderungen des AMNOG-Verfahrens der nächsten Jahre werden. Unabhängig davon könnte es für die Weiterentwicklung des AMNOG-Verfahrens sinnvoll sein, in Orphan Drug-Verfahren eine Orientierung an weiteren Preispunkten wie den Therapiekosten anderer Orphan Drugs in vergleichbar großen (bzw. kleinen) Anwendungsgebieten oder an europaweit verhandelten Höchstbeträgen anzustreben.

\subsection{Ausblick: Neukalibrierung der nutzenbasierten Preisbildung}

Aufgrund der vermehrten Zulassung von Hochpreistherapien bildet sich ein international bereits laufender Diskurs um die Bezahlbarkeit (Affordability Challenge) neuer Therapieverfahren inzwischen auch in Deutschland ab (Hampson et al. 2018). Diskutiert wird, ob es zukünftig weiterer Marktzugangshürden oder ergänzender Regulierungsansätze bedarf, um die Bezahlbarkeit hochpreisiger Arzneimitteltherapien sicherzustellen. Aus gesundheitsökonomischer Perspektive ist dabei die bislang fehlende strukturierte Berücksichtigung ökonomischer Evidenz im Rahmen der Bewertung und Preisfindung zu beklagen. Der Gesetzgeber sieht vielmehr eine starke Orientierung der Preisbildung an dem Ergebnis der frühen Nutzenbewertung sowie den bisherigen Kosten im Therapiegebiet vor, für die Umsetzung dieser Kriterien zur Preisbildung wurden indes nur Rahmenbedingungen vorgegeben. Preise neuer Arzneimittel sollen sich 
damit, sofern ein Zusatznutzen belegt ist, aus einem Verhandlungsverfahren ergeben. Wie diese Kriterien - im Rahmen der Preisbildung durch den GKV-Spitzenverband und die pharmazeutischen Unternehmer - berücksichtigt werden und ob weitere Kriterien einbezogen werden sollen, ist Gegenstand laufender Diskussionen.

Daher erscheint es sinnvoll, die Informationsbasis für das derzeitige Preisbildungsverfahren zumindest optional um zusätzliche Informationen aus Kosten-Nutzen-Bewertungen (anhand ausgewählter Verfahren mit Pilotstudien) zu erweitern. Dafür wäre aufgrund der unsicheren Evidenz und der hohen Kosten ein $\mathrm{ATMP}^{7}$-Verfahren besonders geeignet. Aber auch in Verfahren mit nur marginalen Zusatznutzen wäre der zusätzliche Informationsgehalt aus Kosten-Nutzen-Bewertungen sinnvollerweise $\mathrm{zu}$ evaluieren (Toumi et al. 2017). Dem vorausgehen wird ein Diskussionsprozess der maßgeblichen Institutionen der Selbstverwaltung, der Fachgesellschaften und Professionen, insbesondere aus der Medizin, Gesundheitsökonomie und Ethik über den institutionellen und verfahrenstechnischen Rahmen einer Kosten-Nutzen-Bewertung sowie deren Methodik. Dass zumindest dieser Diskussionsprozess inzwischen vonseiten des IQWiG angestoßen wurde, ist aus gesundheitsökonomischer Perspektive zu begrüßen.

\section{Literatur}

Arznei-Telegramm (2017) Preistreiberei bei Orphan Drugs; In Sieben Jahren um das 450-Fache verteuert. a-t 48:57-58

Ärzteblatt (2017) Kosten für onkologische Arzneimittel steigen massiv. https://www.aerzteblatt. de/nachrichten/76501/Kosten-fuer-onkologischeArzneimittel-steigen-massiv

Beckmann S, Neumann M, Nink K, Selke G (2010) Der Weg zu fairen Pillenpreisen. Gesundh Ges 4:21-27

Bundesministerium für Gesundheit (2020) KJ 1-Statistik, gesetzliche Krankenversicherung: endgültige Rechnungsergebnisse; Dokumentationsstand: 04.05.2020. https://www.bundesgesundheitsministerium.de/

7 Arzneimittel für neuartige Therapien (ATMP, Advanced Therapy Medicinal Products).
fileadmin/Dateien/3_Downloads/Statistiken/GKV/ Finanzergebnisse/KV45_1-4_Quartal_2019_Internet. pdf. Zugegriffen: 21. Sept. 2020

Bundestags-Drucksache (2010) Gesetzentwurf der Fraktionen der CDU/CSU und FDP Entwurf eines Gesetzes zur Neuordnung des Arzneimittelmarktes in der gesetzlichen Krankenversicherung vom 6. Juli 2010 (Arzneimittelmarktneuordnungsgesetz - AMNOG)

Bundestags-Drucksache (2013) Kleine Anfrage der Abgeordneten Kathrin Vogler, Sabine Zimmermann (Zwickau), Harald Weinberg, Birgit Wöllert, Pia Zimmermann und der Fraktion DIE LINKE. Auswirkungen von Herstellerrabatten und Nutzenbewertung auf die Arzneimittelversorgung und -ausgaben; vom 2. Dez. 2013

Busse R, Panteli D, Henschke C (2015) Arzneimittelversorgung in der GKV und 15 anderen europäischen Gesundheitssystemen. Ein systematischer Vergleich. Working papers in health policy and management, Nr. 11. Universitätsverlag der TU Berlin, Berlin

Cassel D, Ulrich V (2016) Das AMNOG auf dem gesundheitsökonomischen Prüfstand. In: Wille E (Hrsg) Entwicklung und Wandel in der Gesundheitspolitik. 20. Bad Orber Gespräche über kontroverse Themen im Gesundheitswesen. Peter Lang, Frankfurt am Main, Bern, Bruxelles, New York, Oxford, Warzsawa, Wien, S 131-160

Coca V, Nink K, Schröder H (2010) Ökonomische Aspekte des deutschen Arzneimittelmarktes 2009. In: Schwabe U, Paffrath D (Hrsg) ArzneiverordnungsReport 2010. Aktuelle Daten, Kosten, Trends und Kommentare. Springer, Berlin, Heidelberg, S 157215

Danzon P (2018) Affordability challenges to value-based pricing: mass diseases, orphan diseases, and cures. Value Health 21:252-257. https://doi.org/10.1016/j. jval.2017.12.018

DiMasi J, Grabowski H, Hansen R (2016) Innovation in the pharmaceutical industry: new estimates of $R \& D$ costs. J Health Econ 47:20-33. https://doi.org/10. 1016/j.jhealeco.2016.01.012

Gerlinger T, Schönwälder T (2012) Arzneimittelversorgung; Wie erfolgte die Umsetzung der Kostendämpfungspolitik mithilfe von verschiedenen Modellen zur Zuzahlung bei Arzneimitteln? https:// www.bpb.de/politik/innenpolitik/gesundheitspolitik/ 72901/arzneimittelversorgung? $\mathrm{p}=$ all

GKV-Spitzenverband (2019) Hochpreisstrategien in der Onkologie. GKV 90 Prozent

Goldstein D, Sarfaty D (2016) Cancer drug pricing and reimbursement: lessons for the United States from around the world. The Oncol 21:907-909. https://doi. org/10.1634/theoncologist.2016-0106

Greiner W (2020) Neustart für die Kosten-NutzenBewertung. Gerechte Gesundh 52

Greiner W, Witte J (2017) AMNOG-Report 2017; Nutzenbewertung von Arzneimitteln in Deutschland. 
Schwerpunkt: Mischpreise. medhochzwei, Heidelberg, Neckar

Greiner W, Witte J (2018) AMNOG-Report 2018; Nutzenbewertung von Arzneimitteln in Deutschland. medhochzwei, Heidelberg

Greiner W, Kuhlmann A, Schwarzbach C (2010) Ökonomische Beurteilung des Effizienzgrenzenkonzeptes. Gesundh ökon Qual manag 15:241-250. https://doi. org/10.1055/s-0029-1245673

Greiner W, Witte J, Gensorowsky D, Pauge S (2020) AMNOG-Report 2020; 10 Jahre AMNOG - Rückblick und Ausblick. medhochzwei, Heidelberg

Hampson G, Towse A, Pearson SD, Dreitlein WB, Henshall C (2018) Gene therapy: evidence, value and affordability in the US health care system. J Comp Eff Res 7:15-28

Hecken J (2019) Regulierung von Arzneimitteln für neuartige Therapien durch den Gemeinsamen Bundesausschuss. GuS 73:8-12. https://doi.org/10.5771/16115821-2019-2-8

Hecken J (2020) Erfolgsmodell AMNOG; Eine Bilanz zehn Jahre nach der Entscheidung. G-BA. https:// observer-gesundheit.de/erfolgsmodell-amnog/. Zugegriffen: 1. Nov. 2020

Hess R (2011) Die Frühbewertung des Nutzens neu zugelassener Arzneimittel. Herausforderungen für den Gemeinsamen Bundesausschuss und das Institut für Qualität und Wirtschaftlichkeit im Gesundheitswesen. GGW 1:8-14

Jablonka S, Bogum J, Opitz G (2021) Ein neuer Weg zu fairen Preisen. G+G digital, 06/2021. https://www.ggdigital.de/2021/06/ein-neuer-weg-zu-fairen-preisen/ index.html

Kifman M, Neelsen S (2010) Germany's struggle with prices for patent-protected drugs. CESifo DICE Report

Korchagina D, Vataire A, Toumi M, Falissard B, Aballéa S (2015) Determinants of orphan drug prices in France: regression analysis. Value Health 18:A337. https:// doi.org/10.1016/j.jval.2015.09.124

Korchagina D, Millier A, Vataire A, Aballea S, Falissard B, Toumi M (2017) Determinants of orphan drugs prices in France: a regression analysis. Orphanet J Rare Dis 12:75. https://doi.org/10.1186/s13023-016-05615

Korzilius H (2019) Arzneimittel: Modelle für gerechtere Preise. Dtsch Arztebl 116:A-503

Laschet H (2020) Unfaire Preise? Disput über Regulation für neue Wirkstoffe; vom 23.09.2020. Ärzte Zeitung online. https://www.aerztezeitung.de/Politik/ Unfaire-Preise-Disput-ueber-Regulation-fuer-neueWirkstoffe-413098.html

Maag G (2010) IMS Marktbericht. Entwicklung des Pharmamarktes im Dezember und Jahr 2009. IMS Health

Maag G (2021) Arzneimittelmarkt 2020 in Deutschland: Innovationen und Auswirkungen der Pandemie im Fokus. IQVIA. https://www.iqvia.com/-/media/ iqvia/pdfs/germany/news/iqvia-arzneimittelmarkt- deutschland-2020-pm-2021-02.pdf?la=de-de\&hash= C04933F63B24EAD63FB9067AF424DA0C

McGrail S (2020) Is Germany's drug pricing strategy an option for the US? https://pharmanewsintel.com/ news/is-germanys-drug-pricing-strategy-an-optionfor-the-us. Zugegriffen: 2. Nov. 2020

Morgan S, Bathula H, Moon S (2020) Pricing of pharmaceuticals is becoming a major challenge for health systems. BMJ 368:14627. https://doi.org/10. 1136/bmj.14627

o. V. (2013) Kassen wollen Margen drücken. Pharmazeutische Zeitung online

o. V. (2020a) Hochpreisige Arzneimittel befeuern Diskussion um Kosten-Nutzen-Bewertung. Ärzteblatt

o. V. (2020b) Too Much? Neuartige Therapien in der Gesetzlichen Krankenversicherung. Gerechte Gesundheit

Picavet E, Morel T, Cassiman D, Simoens S (2014) Shining a light in the black box of orphan drug pricing. Orphanet J Rare Dis 9:1-10. https://doi.org/10.1186/ 1750-1172-9-62

Sachverständigenrat zur Begutachtung der Entwicklung im Gesundheitswesen (2014) Bedarfsgerechte Versorgun; Perspektiven für ländliche Regionen und ausgewählte Leistungenbereiche

Schlander M, Jäcker A, Völkl M (2012) Preisbildung in einem besonderen Markt. Ärzteblatt 109:A524-A528

Simoens S (2011) Pricing and reimbursement of orphan drugs: the need for more transparency. Orphanet J Rare Dis 6:1-8. https://doi.org/10.1186/1750-1172-6-42

Sorenson C, Drummond M, Kanavos P (2008) Ensuring value for money in health care; The role of health technology assessment in the European Union. World Health Organization on behalf of European Observatory on Health Systems and Policies, Geneva

Stegmaier P (2019) Es geht um Ideen und nutzenstiftende Grundprinzipien; Interview mit Prof. Dr. Volker Amelung, Hans-Holger Bleß und Ralph Lägel. Monit Versorgungsforsch 04:16-19

Sussell J, Vanderpuye-Orgle J, Vania D, Goertz H, Lakdawalla D (2019) Understanding price growth in the market for targeted oncology therapies. Am J Manag Care 5:273-277

Sussex J, Towse A, Devlin N (2013) Operationalizing value-based pricing of medicines : a taxonomy of approaches. PharmacoEconomics 31:1-10. https://doi. org/10.1007/s40273-012-0001-x

Toumi M, Motrunich A, Millier A, Rémuzat C, Chouaid C, Falissard B, Aballéa S (2017) Analysis of health economics assessment reports for pharmaceuticals in France - understanding the underlying philosophy of CEESP assessment. J Mark Access Health Policy 5:1344088. https://doi.org/10.1080/20016689. 2017.1344088

Wasem J (2018) Was ist der Gesellschaft der Zusatznutzen wert? Gerechte Gesundh 41 
Open Access Dieses Kapitel wird unter der Creative Commons Namensnennung 4.0 International Lizenz (http:// creativecommons.org/licenses/by/4.0/deed.de) veröffentlicht, welche die Nutzung, Vervielfältigung, Bearbeitung, Verbreitung und Wiedergabe in jeglichem Medium und Format erlaubt, sofern Sie den/die ursprünglichen Autor(en) und die Quelle ordnungsgemäß nennen, einen Link zur Creative Commons Lizenz beifügen und angeben, ob Änderungen vorgenommen wurden.

Die in diesem Kapitel enthaltenen Bilder und sonstiges Drittmaterial unterliegen ebenfalls der genannten Creative Commons Lizenz, sofern sich aus der Abbildungslegende nichts anderes ergibt. Sofern das betreffende Material nicht unter der genannten Creative Commons Lizenz steht und die betreffende Handlung nicht nach gesetzlichen Vorschriften erlaubt ist, ist für die oben aufgeführten Weiterverwendungen des Materials die Einwilligung des jeweiligen Rechteinhabers einzuholen.

(c) (1) 